\title{
Elementary Considerations of the Effects of Multipath Propagation in Meteor-Burst Communication
}

\author{
George R. Sugar, Robert J. Carpenter, and Gerard R. Ochs
}

(April 14, 1960; revised April 25, 1960)

\begin{abstract}
Three mechanisms likely to regularly produce multipath propagation are examined. These are: (1) The simultaneous existence of two meteor trails; (2) the existence of a Rayleigh-fading background continuum; and (3) the existence of two first-Fresnel zones along a single meteor trail.

An analysis of the first mechanism indicated that in a typical meteor-burst communication system two-trail propagation would cause transmission errors at a rate directly proportional to the system duty cycle. Satisfactory agreement was obtained between predicted and observed error rates for such a system. An examination of the significance of interference from the continuum in some wide-band transmission tests indicated that this source of multipath could be responsible for a significant fraction of the errors observed. The third mechanism was examined to determine the magnitude of the multipath delays it could produce. It was found that the effect of this single-trail multipath was likely to be significant only for transmission rates in excess of $2 \times 10^{4}$ bauds. However, the results of measurements at a rate of $10^{5}$ bauds indicated that even at this high rate over one-half of the transmissions were error free and that this latter type of multipath may not be of much importance in system design.
\end{abstract}

\section{Introduction}

One of the important results of the first theoretical studies of meteor-burst communication $[1,2]^{2}$ was that the average channel capacity could be increased by raising the signaling rate. It is likely that this result, more than any other, led to the extensive study and development of meteor-burst systems. However, when experimental systems were tested it was found that the channel capacity observed fell far short of that predicted by theory. (For example, in one set of measurements [3], at a signaling rate of $10^{5}$ bauds the predicted channel capacity was $10^{4}$ bauds at an error rate of $10^{-5}$. The measured capacity, however, was $10^{3}$ bauds at an error rate near $10^{-3}$.) This discrepancy between theory and experiment has led to a search for ways of modifying or extending previous theoretical results so that practical channel capacities could be accurately predicted. One aspect under study at NBS has been an examination of the deleterious effects of multipath propagation.

Multipath propagation is one source of transmission error and this fact, although recognized, was neglected in the development of the meteor-burst communication theory. In this paper three likely sources of multipath propagation will be considered. The first arises from the possibility that two meteor trails can exist simultaneously. The second arises because a secondary signal is propagated by the background continuum (the so-called "ionospheric scatter" mode of propagation). The third situation

1 Contribution from Centra Radio Propagation Laboratory, National Bureau of Standards, Boulder, Colo.

${ }_{2}$ Figures in brackets indicate the literature references at the end of this paper. to be considered arises as a consequence of the existence of single long-enduring meteor trails which have been distorted by winds in the ionosphere.

It has not been practical, as yet, or even desirable to consider the practical effects of these three classes of multipath propagation from a unified viewpoint. In each case the analysis has been carried only to the point where some results of practical value were obtained, and no attempt has been made to consider the effects in combination rather than separately.

For the two-trail case a general relation between error rate, duty cycle, and the statistical parameters of meteor-burst propagation is developed and then applied to a hypothetical fsk communication system. In the case of multipath interference from the continuum the interference is treated as being a noiselike signal which reduces the effective signal-to-noise ratio at the receiver. ${ }^{3}$ For the case of multipath propagation from a single trail the analysis is limited to a computation of the maximum delay which is likely to be produced. The application of the computational techniques is illustrated by using them to compute error rates for two experimental meteorburst communication systems and comparing these with the error rates actually observed.

No consideration is given to the frequentlyobserved signal fading which can result from quite short multipath delays, since the equipment techniques for combating such fading are well known and readily available. Consideration is limited instead to those multipath conditions which are likely to result in receiving strong but badly-distorted signals for systems utilizing conventional modulation techniques such as fsk or ppm.

3 This is similar to the treatment by Montgomery and Sugar [1] 


\section{Two-Trail Multipath Propagation}

The binary error rate for a meteor-burst system is now computed under the assumption that the only source of error is the multipath propagation which results from the simultaneous existence of two suitably-oriented meteor trails. Three conditions must be met. First, there must be two trails present at the same time with the amplitude of the signal from one of them above the operating threshold of the system. Second, the signal from the second trail, the interfering signal, must be comparable in amplitude to that from the first trail. Third, the interfering signal must be sufficiently delayed so as to cause errors (rather than just causing fading). The exact details of the latter two requirements will depend on the characteristics of the particular communications system under study. The error for an fsk radiotelegraph system is computed since experimental results from two such systems are available for comparison with the theory. However, the same general procedure can be followed in predicting the error for any system.

Assume that meteoric particles enter the earth's atmosphere at random times and that the radio properties of trails are statistically independent of the time when the trail was formed. Let the distribution function $F(S)$ represent the probability that the signal amplitude is greater than $S$, and let $f(S)$ represent the associated probability density. Then for a system threshold $S_{t}$ the duty cycle or probability that the signal will be above $S_{t}$ is just $F\left(S_{t}\right)$. The probability that signals from trails 1 and 2 will be simultaneously present and contribute amplitudes $S_{1}$ and $S_{2}$ respectively is

$$
f\left(S_{1}\right) f\left(S_{2}\right) d S_{1} d S_{2} .
$$

Assume that the error rate for a binary signaling system can be given by a function $p\left(S_{1}, S_{2}, \tau\right)$ where $\tau$ is the multipath delay. Thus the error for signals $S_{1}, S_{2}$, and multipath delay $\tau$ is

$$
p\left(S_{1}, S_{2}, \tau\right) f\left(S_{1}\right) f\left(S_{2}\right) d S_{1} d S_{2} d \tau .
$$

The average error rate $P$ for the interval $T$ is then given by

$$
\begin{aligned}
& P\left(S_{t}\right) \\
& =\frac{\int_{\tau=0}^{\infty} \int_{S_{1}=S_{t}}^{\infty} \int_{S_{2}=0}^{S_{1}} p\left(S_{1}, S_{2}, \tau\right) f\left(S_{1}\right) f\left(S_{2}\right) d S_{1} d S_{2} d \tau}{F\left(S_{t}\right)} .
\end{aligned}
$$

$\left(S_{1}\right.$ is chosen here as the amplitude of the stronger signal and it is assumed that during each binary digit $S_{1}, S_{2}$, and $\tau$ are constant.) It remains now only to put in the functions $p$ and $f$ for a given system and evaluate the integral. Since experimental data for two fsk radiotelegraph systems is available the error will be computed for such a system.

Assume that all signals result from specular reflections from underdense trails, that the decay param- eter $k$ (in $S=S_{\max } e^{-k t}$ ) is fixed over the observing interval, that the system uses fsk and that the receiver has an effective capture ratio $R$. ( $R$ is defined as the amplitude ratio of the stronger signal to the weaker signal required to achieve error-free reception.) Then

$$
P\left(S_{t}\right)=\frac{\int_{S_{1}=S_{t}}^{\infty} \int_{S_{2}=0}^{S_{1}} \frac{1}{2} f\left(S_{1}\right) f\left(S_{2}\right) d S_{1} d S_{2}}{F\left(S_{t}\right)}
$$

if $1<S_{1} / S_{2}<R, \tau>\tau_{0}$, and $P\left(S_{t}\right)=0$ otherwise. If the distribution function is assumed to be given by $F(S)=\left(S_{0} / S\right)^{a}$ where $S_{0}$ and $a$ are constants, then $f(S)=(a / S)\left(S_{0} / S\right)^{a}$.

For the present assume that any multipath delay is sufficient to cause system errors. ${ }^{4}$ This implies that $\tau_{0}=0$ and

$$
P\left(S_{t}\right)=\frac{\int_{S_{1}=S_{t}}^{\infty} \int_{S_{2}=S_{1} / R}^{S_{1}} \frac{a^{2}}{2} S_{0}^{2 a} S_{1}^{-a-1} S_{2}^{-a-1} d S_{1} d S_{2}}{F\left(S_{t}\right)} .
$$

After integration the result becomes

$$
P\left(S_{t}\right)=\frac{1}{4}\left(R^{a}-1\right)\left(S_{0} / S_{t}\right)^{a} .
$$

Then, since the duty cycle for any threshold is just

$$
F\left(S_{t}\right)=\left(S_{0} / S_{t}\right)^{a},
$$

in terms of duty cycle

$$
P\left(S_{t}\right)=\frac{1}{4}\left(R^{a}-1\right) F\left(S_{t}\right) .
$$

The error rate is therefore proportional to duty cycle. Figure 1 is a plot of the factor $\frac{1}{4}\left(R^{a}-1\right)$ for various values of the parameters $R$ and $a$.

Some results obtained from the NBS MeteorBurst Communication System [5] are now compared with those predicted by the above theory. The operating data available for the system gives character error rate and duty cycle as a function of the system parameters. The first step is to relate binary error to character error. When the system is operated at low error rates, it is estimated that each binary transmission error will produce an average of 1.35 character errors on the teleprinter. (This estimate should not be considered generally applicable to other radio-teletype systems since it is based on a specific set of rules for counting errors and also includes a consideration of control errors in the system.) Through the use of this factor the character error rate can be predicted and compared with the measured error rate. This has been done for various values of $R$ and the comparison is shown in figure 2 . Each point represents the average error rate and duty cycle for $1 / 2 \mathrm{hr}$ of system operation.

4 At present some data is available which can be interpreted to give multipath delays. Bailey, Bateman, and Kirby [4] give delays of meteor bursts relative to the continuum at $49.7 \mathrm{Mc} / \mathrm{s}$ and these can be interpreted as indicating that the most probable multipath delay in the two-trail case is of the order of $200 \mu$ sec or less. Delays up to $2 \mathrm{msec}$ were observed occasionally. 


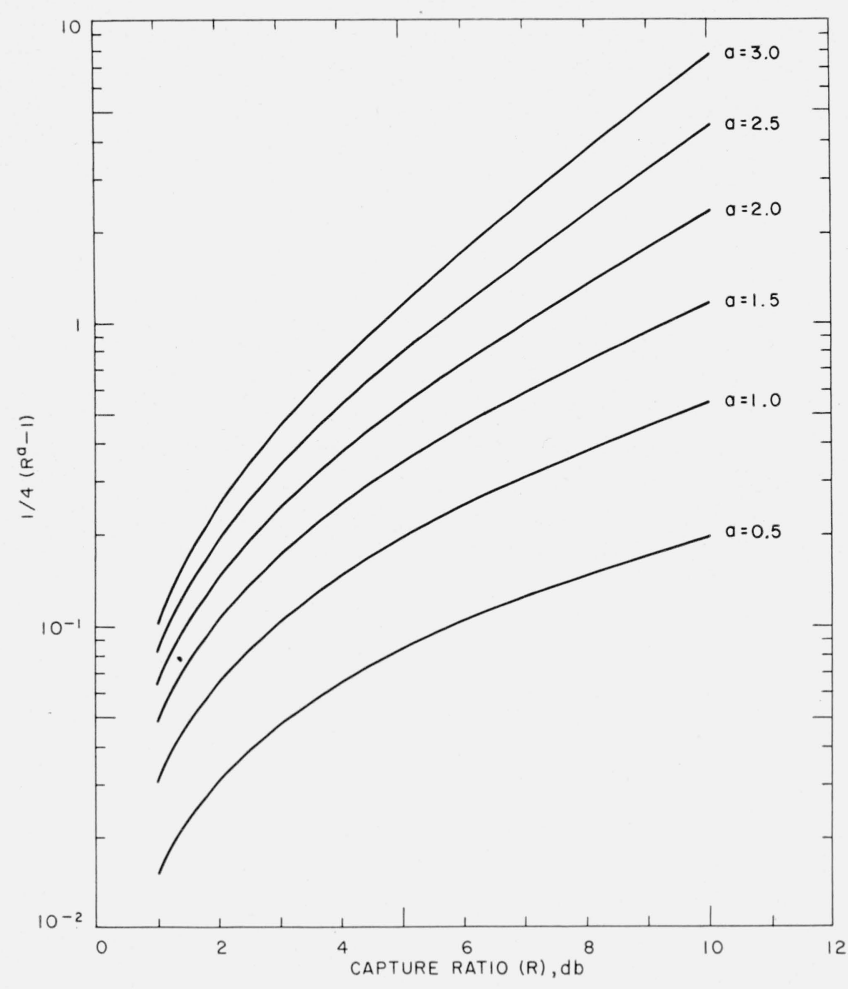

Figure 1. Error rate factor for fsk transmission. Error rate $=$ ordinate $\times$ duty cycle.

Unfortunately, the capture ratio $R$ was not measured for the system and an exact comparison between predicted and observed error rates is not possible. However, measurement in similar systems has indicated that capture ratios of 2 to $3 \mathrm{db}$ are readily attainable. It therefore appears that the theoretically predicted results are not inconsistent with the observations and there may in fact be good agreement. The slope of the experimental data is quite close to that predicted and the magnitude is representative of that predicted for a system with an effective capture ratio of 3 to $4 \mathrm{db}$.

\section{Multipath Interference From the Continuum}

The effect of interference from the continuous background signal on an idealized meteor-burst communication system is now considered and the predicted error rates are compared with some experimental results. For the burst system itself, errorfree performance in the absence of the continuum is assumed. In addition it is assumed that all of the multipath delays involved are at least comparable to the bit length and therefore can cause transmission errors. The signal from the continuum can then be considered as being a type of Gaussian noise which sets the effective signal-to-noise ratio for the system. This assumption is justified since the continuum exhibits a Rayleigh fading characteristic [6]. Moreover for large signaling bandwidths the continuum signal is not phase coherent over the

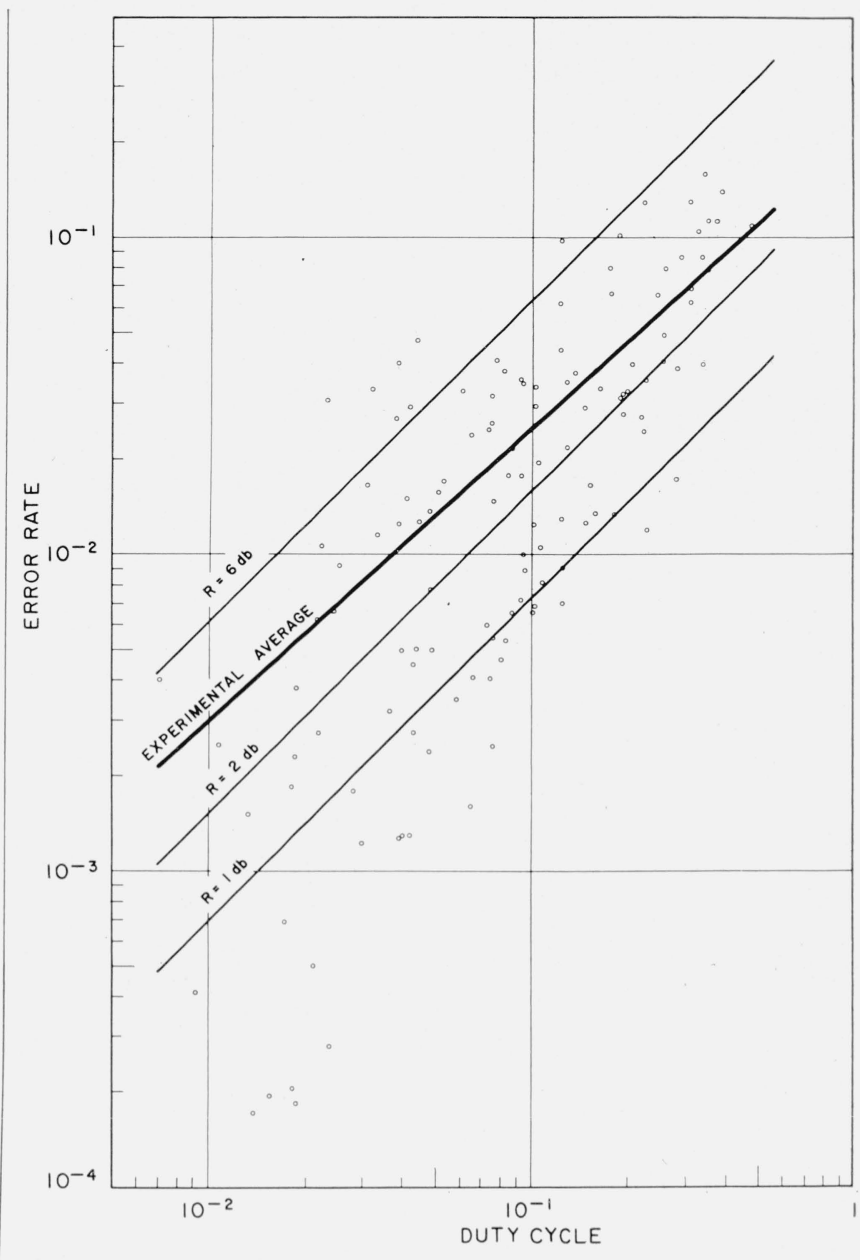

FIgure 2. Computed and observed error rates for two-trail multipath.

The 3 light lines give computed error rates. The points and heavy line are experimental data.

band $[4,7]$. Thus to a first approximation the continuum signal is not correlated with the meteorburst signal and can therefore be analyzed as if it were random noise.

Under these assumptions, the error rate for a system can readily be predicted since the signal-tonoise-ratio performance of most modulation systems has been worked out and is available in the literature. For simplicity the signal level can be taken to be the signal threshold at which the burst system is operated. The more complex procedure of using the actual meteoric signal distribution could be followed, but at present there seems to be little to be gained by using this more difficult approach since even it is highly idealized and neglects many restrictions present in actual systems.

For a comparison between theory and experiment the results from some wide-band meteor-burst transmission studies performed at NBS ${ }^{5}$ are utilized. The signaling rate was $10^{5}$ bauds using fsk, and

${ }^{5}$ These studies were a continuation of the work reported by Montgomery and Sugar [1]. 
binary error was measured. The continuum was measured during the experiment by use of a stripchart recorder operated from the agc circuits of the fsk receiver. For the theoretical relation between error and signal-to-noise ratio, that given by Montgomery [8] for fsk is used. This is plotted in figure 3, as the curve labelled $0 \mathrm{db}$, along with the experimental data. Each small circle represents the error rate observed over a 15-min interval. (The large circles represent averages of the 15 -min points. The points plotted with an $x$, the apparatus capability, indicate the performance of the equipment as measured in the laboratory using a nonfading signal and Gaussian noise. The additional curves indicate the performance to be expected if the system is 5,10 , or $15 \mathrm{db}$ poorer than the theoretical prediction.) It is seen that at high error rates the experimental results fall within a few decibels of the predicted values. However, at low error rates the agreement is poor and there are discrepancies of up to $15 \mathrm{db}$ between theory and experiment. These observations are interpreted as indicating that at high error rates the observed error can almost wholly be accounted for in terms of multipath interference from the continuum whereas at low error rates some other sources of error are controlling. It is useful to now consider two-trail multipath as another possible source of error here.

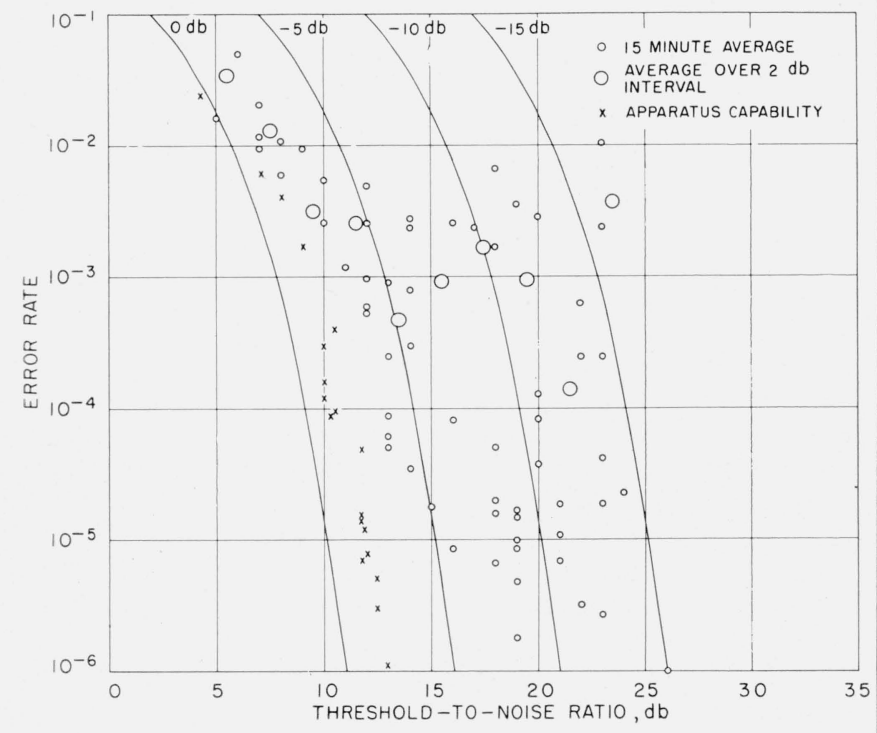

Figure 3. Computed and observed error rates for a system subjected to multipath interference from the continuum.

The apparatus capability as indicated in figure 3 is about $2 \mathrm{db}$ poorer than the theoretical prediction. It is therefore assumed that this represents a receiver capture ratio of $2 \mathrm{db}$. For the distribution we take the value $a=1.2$ as used before. It is found then from figure 1 that the predicted error rate is 0.08 times the duty cycle. When this is applied to the data given in figure 3 , it is found that the predicted error rate varies from about $10^{-4}$ for low threshold-tonoise ratio data to about $10^{-5}$ for high threshold-tonoise ratio data. The observed error rates are 10 to 200 times these values. Thus it appears that twotrail multipath does not contribute significantly to the observed error rate, and therefore, that still another source of error is important.

\section{One-Trail Multipath Propagation}

The last possibility to be considered is that of having two distinct transmission paths result from the entry of a single meteoric particle into the earth's atmosphere. This will occur when, through the action of wind shears in the ionosphere, a single, overdense trail becomes distorted into an irregular column and as a result has two (or more) "first" Fresnel zones. These could be separated as much as the whole length of the trail and thus produce two distinct transmission paths. The multipath delays to be expected from this mechanism are, as will be shown, quite small and therefore of importance only in very high signaling rate systems. The computation of error for this case appears to be substantially more complex than either of the two previous cases and we have not attempted it. However, some of the factors involved in such a computation can be indicated.

First consider the magnitude of the multipath delay. It has been shown that for a trail which originally gives a specular reflection, the action of the wind shears is to produce "glints" (local first Fresnel zones) which first form near the original first Fresnel zone and later form further out along the trail $[9,10]$. The maximum delay results when there is a glint at each end of the trail, and therefore the delays produced by two glints separated by a distance $L$ are computed. Several trail orientations are possible and for each of these a different delay will result from a given $L$. The limiting cases are given below. (All of these are for long oblique paths with one end of the trail formed at the path midpoint.)

(a) Trail normal to plane of propagation

$$
\begin{aligned}
& \text { delay } \sim L^{2} /(c D) \\
& \text { where } L=\text { glint separation, } \\
& D=\text { slant range to path midpoint, } \\
& \text { and } \\
& c=\text { velocity of propagation. }
\end{aligned}
$$

(b) Horizontal trail in the plane of propagation

$$
\begin{aligned}
& \text { delay } \sim\left(L^{2} \cos ^{2} \theta\right) /(c D) \\
& \text { where } \theta \text { is } 1 / 2 \text { the angle between the } \\
& \text { incident and reflected rays at the } \\
& \text { path midpoint. }
\end{aligned}
$$

(c) Vertical trail in the plane of propagation ${ }^{6}$

$$
\text { delay } \sim(2 L \cos \theta) / c .
$$

6 Trails oriented in this manner are of minor practical imnortance. They do not have first Fresnel zones at the time of formation and will have glints only if they endure long enough to be severely distorted by wind shears. 
For trail lengths the values published by Eshleman [11] are taken. These data indicate that the most probable trail length is about $15 \mathrm{~km}$, with trails 40 to $50 \mathrm{~km}$ long occurring about 5 percent of the time. (The ends of a trail are defined here as being the points with a specific electron density.) As the limiting case, it is assumed that the separation $L$ between glints is equal to the trail length. (The delays experienced in any experiment would be expected to be less than those predicted by this method.) Table 1 gives the delays predicted for the three cases. These delays are likely to produce transmission distortion in fsk systems operating at speeds of 20,000 bauds or greater.

TABLE 1. Maximum multipath delay expected from an irregular overdense trail

$$
(D=1277 \mathrm{~km})
$$

\begin{tabular}{|c|c|c|}
\hline \multirow[t]{2}{*}{ Trail orientation } & \multicolumn{2}{|c|}{ Trail length } \\
\hline & $15 \mathrm{~km}$ & $40 \mathrm{~km}$ \\
\hline $\begin{array}{l}\text { Normal to propagation plane } \\
\text { Horizontal, in plane } \\
\text { Vertical, in plane }\end{array}$ & $\begin{array}{l}11 \mu \mathrm{S} \\
0.5 \\
20\end{array}$ & $\begin{array}{l}82 \mu \mathrm{S} \\
3.4 \\
54\end{array}$ \\
\hline
\end{tabular}

It would be desirable to calculate error rate in this case as was done in section 2. However, we see no straightforward way to do so. Consideration of the error associated with overdense trails requires that we know the distribution of glint separations, how these are related to the length of the meteor trail, and the relative contribution of overdense trails to the total channel capacity. The latter, of course is closely associated to system design parameters such as sensitivity, operating frequency, antenna directivity, and control system design philosophy. Since the signal from a specular overdense trail is likely to exhibit fading long before producing transmission distortion, a system can be arranged to stop signaling before any significant delays can occur, and thus this case may be of little practical importance. For the nonspecular overdense case, again similar data on burst statistics are required and these are not available. Therefore we have stopped here with only a computation of delays and hope to extend this work at a later time.

\section{Discussion}

As is often the case there is a significant question whether the experimental results presented were taken under the conditions assumed, implicitly or explicitly, in the theory. Some discussion of this point seems desirable.

In the two-trail case, it was assumed that multipath distortion was the only source of error and satisfactory agreement was obtained between theory and experiment. One can ask, however, if the same experimental result would have been obtained even if two-trail multipath were not present. This result might be possible if all errors resulted from noise interference instead of multipath. The data plotted in figure 2 were taken at several thresholds and thus might reflect primarily the effect of changing the threshold-to-noise ratio rather than any multipath phenomena. This does not appear to be the case, however, since data for a single threshold show the same relation as that shown in figure 2 , and it is concluded that the correlation between theory and experiment is meaningful and not fortuitous. A second question arises in regard to the scatter of data in figure 2 both above and below the theoretical curve. It could be argued that the observed error rate should always be greater than the predicted value (as indeed it was in fig. 3) since some sources of error have been ignored. However, this does not seem to be a valid objection when we consider that the data for figure 2 were taken from an operating communication system in which a variety of special techniques were being used to stop transmissions at times when high error rates were likely. This control of transmission will, of course, bias the results so that error rates less than those predicted will be observed. (No such devices were used in the system represented by the data of fig. 3.)

A third question to ask is why the second kind of multipath distortion was not considered in relation to the data shown in figure 2 , and was considered in relation to figure 3 . The difference here lies in the difference in antenna systems used in the two experiments. In the NBS Meteor-Burst Communication System, the antennas were arranged with a null along the great circle path and data were taken during normal working hours. This results in the suppression of the continuum relative to the meteoric bursts and nearly all of the data shown in figure 2 correspond to threshold-to-noise ratios of $12 \mathrm{db}$ or greater. It therefore seems reasonable to ignore the effect in this case. For the $10^{5}$-baud measurements, however, the situation was reversed, for the antennas were high-gain rhombics directed along the greatcircle path. This, of course, enhances the continuum and suppresses meteoric burst signals. Thus, in the second case the continuum was of greater importance.

A final item for discussion is the total effect of the various kinds of multipath distortion on error rate in high rate systems. Data from the $10^{5}$-baud system are plotted in figure 4 so as to show the relation between the information capacity and error rate for single bursts. Each point represents one transmission. The data have been divided into class intervals and are plotted opposite the lower bound of the interval. (In the experiment the data were transmitted in groups of 4,000 binary digits, each group lasting $1 / 25$ sec.) Data for thresholdto-noise ratios less than $12 \mathrm{db}$ have not been plotted since it is unlikely that an fsk system would be operated in that region. It can be seen from figure 4 that many transmissions are error-free and that there is a tendency for the longer transmissions to have higher error rates than the shorter ones. The data show that 57 percent of the transmissions, carrying 32 percent of the channel capacity, are error-free. 


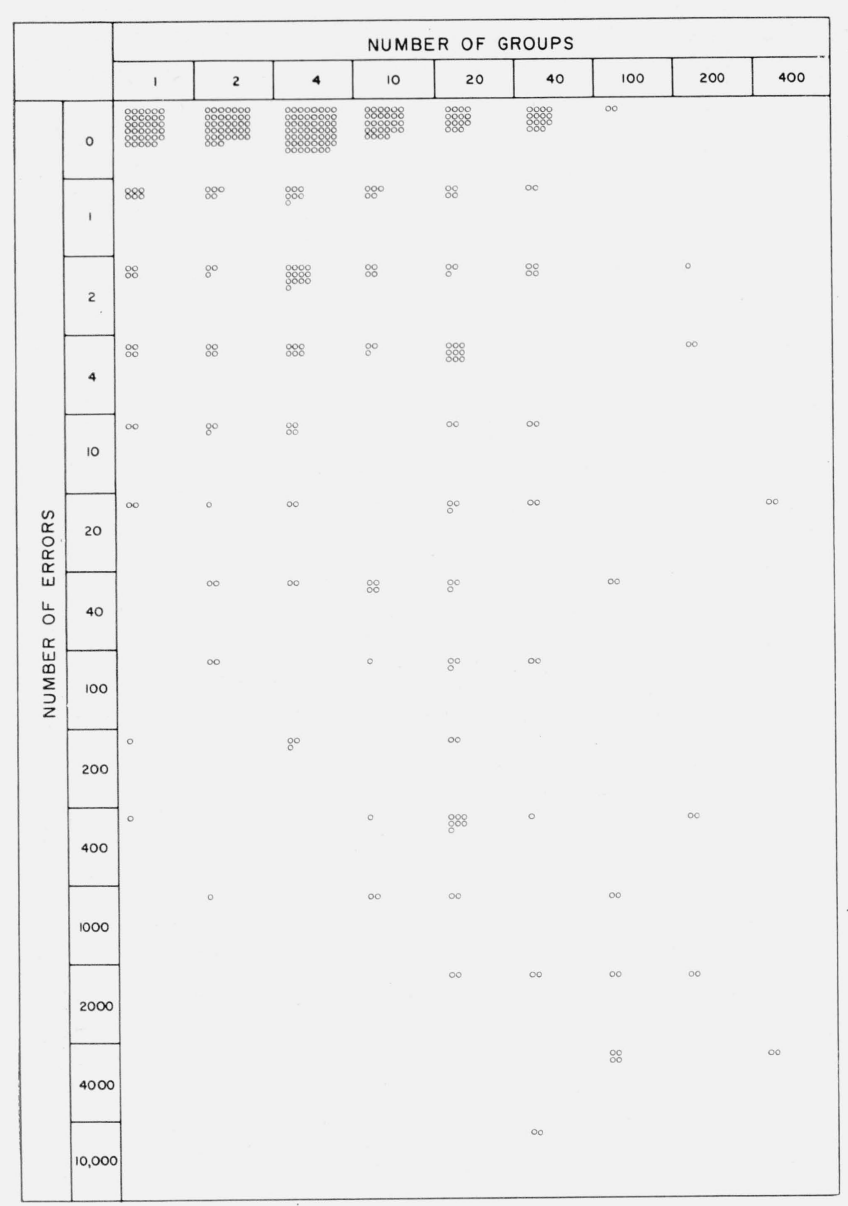

Figure 4. Errors observed for individual transmissions in a $10^{5}$-baud fsk system.

Each point gives the transmission length and number of errors observed. (One group equals 4,000 binary digits.)

For 86 percent of the transmissions, carrying 64 percent of the channel capacity, the error rate is less than $10^{-3}$. It therefore appears that a signaling rate of $10^{5}$ bauds is not so high as to be rendered useless by transmission distortion. Whether or not such systems will have the practical utility of low rate systems will depend on the ingenuity of the designing engineers.

\section{Summary}

Some aspects of multipath transmission and the transmission distortion which can result from it have been considered. For the two-trail case, the relation between error rate and duty cycle for fsk transmission have been computed. In applying this result to a specific system, fair agreement is found. However, there are a number of known factors which would be expected to increase the spread of the experimental data. The agreement is considered satisfactory, and it is concluded that the assumptions involved are reasonably valid and applicable to the specific system examined.
For the second case, the interference from the continuum was examined, and error rate was computed assuming that the interference could be considered as being of the nature of random noise. When the result was compared with data from the $10^{5}$-baud system (one where the assumptions seemed applicable), good agreement between theory and experiment for low values of threshold-to-continuum ratio was found. At high values of this ratio, the observed error rates were 10 to 200 times higher than would be predicted from either two-trail or continuum-multipath theory.

The multipath delays expected from a single trail were estimated to be as great as $82 \mu$ sec and these delays may account for the above discrepancy. However, insufficient data are available to permit any reasonable estimate of the likelihood of error arising from this source.

Some overall results of the $10^{5}$-baud transmission experiment were examined, and these indicated that most of the individual transmissions had low error rates and that these low-error-rate bursts accounted for more than half of the signaling capacity of the system. It therefore appears that signaling rates of $10^{5}$ bauds or higher can be used by devising special circuitry to reject (or repeat) transmissions having high error rates

\section{References}

[1] G. F. Montgomery and G. R. Sugar, The utility of meteor bursts for intermittent radio communication, Proc. IRE 45, 1684 (1957).

[2] L. L. Campbell and C. O. Hines, Bandwidth consideration in a JANET system, Proc. IRE 45, 1658 (1957).

[3] G. F. Montgomery and G. R. Sugar, unpublished report, National Bureau of Standards, September 1955. Most of this material has been published [1].

[4] D. K. Bailey, R. Bateman, and R. C. Kirby, Radio transmission at VHF by scattering and other processes in the lower ionosphere, Proc. IRE 43, 1181 (1955). See fig. 63.

[5] R. J. Carpenter and G. R. Ochs, The NBS meteor-burst communication system, IRE Trans. PGCS CS-7, 263 (1959)

[6] G. R. Sugar, Some fading characteristics of regular VHF ionosphere propagation, Proc. IRE 43, 1432 (1955).

[7] J. W. Koch, Factors affecting modulation techniques for VHF scatter studies, IRE Trans. PGCS CS-7, 77 (1959).

[8] G. F. Montgomery, A comparison of amplitude and angle modulation for narrow-band communication of binary-coded messages in fluctuation noise, Proc. IRE 42, 447 (1954).

[9] L. A. Manning, Air motions and the fading, diversity, and aspect sensitivity of meteoric radio echoes, J. Geophys. Research 64, 1415 (1959).

[10] J. S. Greenhow and E. L. Neufeld, Turbulence at altitudes of $80-100 \mathrm{~km}$ and its effects on long-duration meteor echoes, J. Atmospheric and Terrest. Phys. 16, 384 (1959)

[11] V. R. Eshleman, The theoretical length distribution of ionized meteor trails, J. Atmospheric and Terrest. Phys. 10, 57 (1957). 